\title{
SIMPLIFICATION OF LAMINAR BOUNDARY LAYER EQUATIONS
}

\author{
Karlo T. Raić * \\ University of Belgrade, Faculty of Technology and Metallurgy
}

Received 23.03.2018

Accepted 15.06.2018

\begin{abstract}
The laminar boundary layer theory has been involved in two domains of transport phenomena: (i) steady-state flow (via Blasius eq.) and (ii) unsteady state flow and/or nonflow (via Newton, Fourier and/or Fick's equations). Listed partial differential equations with the similarity of solutions enable the substitution of the observed phenomena by only one-second order differential equation. Consequently, an approach established on the general polynomial solution is described. Numerical verification of the concept is presented. Experimental notifications are documented. Finally, the new simulation strategy is suggested.
\end{abstract}

Keywords: materials processing simulation; laminar boundary layer; second order differential equation; polynomial solution.

\section{Introduction}

Nowadays it is mostly clear, that diverse experiments and events which appeared under various laminar boundary layers [1-3] need new easy-to-use, inexpensive and accurate simulation strategy.

Partial differential equations usually define the laminar transport phenomena. The techniques such as methods of combination of variables and/or separation of variables, a method of sinusoidal response, integral methods, etc., which transform the solving of the partial differential equation into a problem of solving one or more ordinary differential equations are well defined [4-7]. Here, the nearby strategy has been enforced but in the more general way.

The starting idea for this approach [8] was taken from well known: (i) Prandtl's two theories regarding the laminar film adjacent to a solid boundary ( $1^{\text {st }}$ the fluid touching the solid boundary has zero velocity with respect to the wall, and $2^{\text {nd }}$ a layer of fluid adjacent to the solid boundary moves in a laminar motion), as well as (ii) simple von Kármán integral method [5-7].

\footnotetext{
* Corresponding author: Karlo T. Raić, karlo@tmf.bg.ac.rs
} 
The generally known problem in the application of integral methods is the definition of satisfactory distribution for velocity, temperature and/or concentration [6, 7]. The corresponding situation commonly limits the certain distribution. When the terms are changed, we need more suitable distribution. To avoid this obstacle, the second order differential equation with the general polynomial solution is proposed in this paper.

The original form of the presented equation was given in 1992 [8]. Over time, this equation has been refined so that its final shape was established in 1995 [9]. In the last 20 years, this equation has been applied to experiments in the field of materials science and engineering [8-13]. Finally, this article presents the complete strategy for solving the governing laminar boundary layer equations as Blasius, Newton, Fourier, and Fick, that are usually used for materials processing simulation.

\section{Mathematical formulation}

As indicated in the literature [7], the boundary layer theory could be applied in two domains of transport phenomena: steady-state flow (via Blasius eq.) and unsteady state flow and/or nonflow (via Newton, Fourier and/or Fick's equations.). These partial differential equations with the similarity of solutions enable the substitution of the observed phenomena by only one-second order differential equation, Fig. 1.

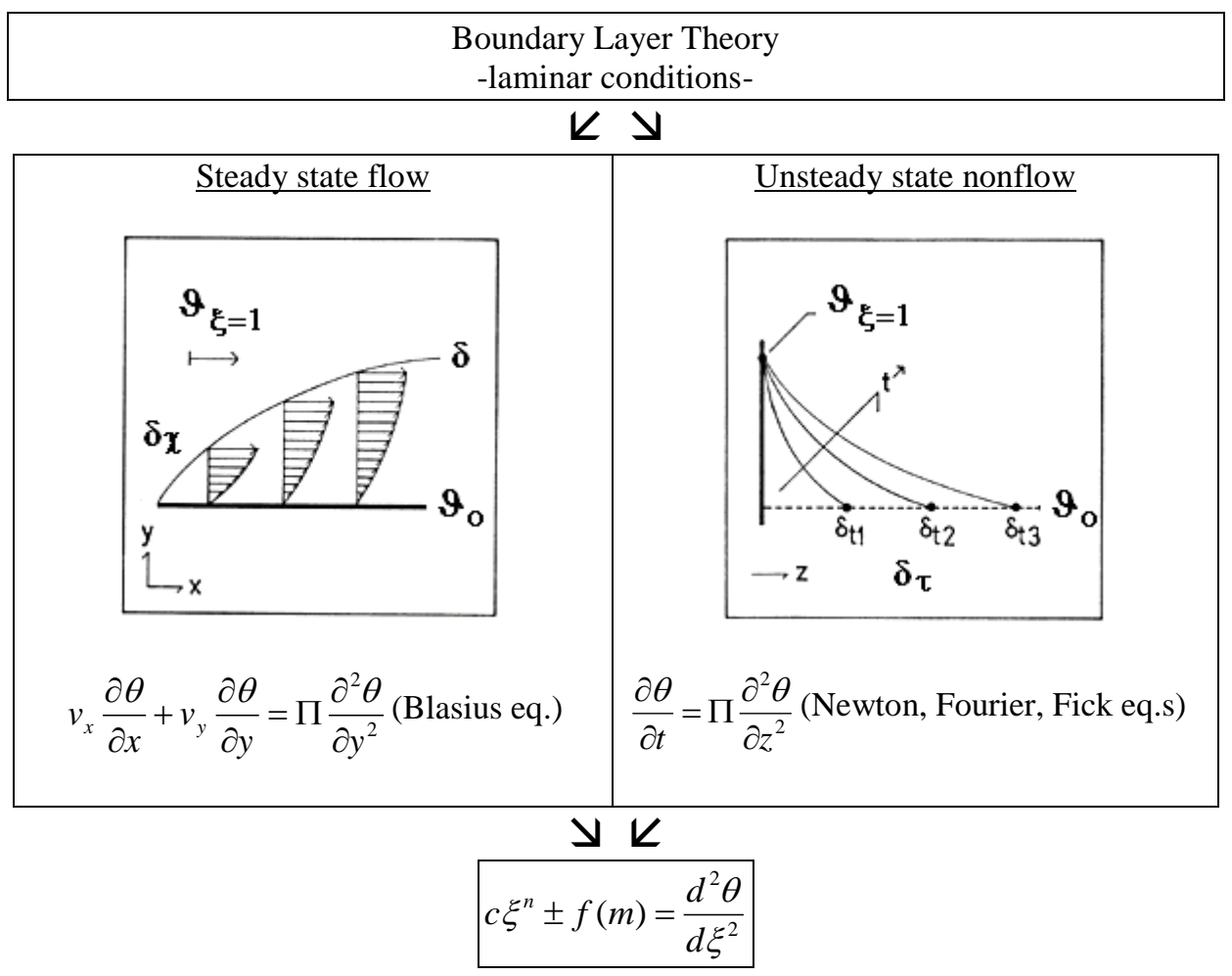

Fig. 1. The concept of substitution of laminar boundary-layer equations under laminar conditions by an ordinary differential equation with the general polynomial solution. 
Proposed second order of ordinary differential equation can be expressed as:

$$
\theta^{\prime \prime}(\xi) \pm c \xi^{n} \pm f(m)=0
$$

where:

$\theta=\frac{\vartheta-\vartheta_{0}}{\vartheta_{\xi=1}-\vartheta_{0}}:$ normalized dimensionless change of quantity $\vartheta$ (velocity, v; temperature, $\mathrm{T}$; and/or concentration, $\mathrm{x}$ );

$\xi=\mathrm{y} / \delta_{\chi}$ or $\xi=\mathrm{z} / \delta_{\tau}$ : dimensionless distance from sur/interface for position $\chi$ or time $\tau$

$\Pi$ : viscosity/diffusivity $(v, \mathrm{a}, \mathrm{D})\left[\mathrm{m}^{2} / \mathrm{s}\right]$;

Surface Criterion: $N=\left.\frac{d \theta}{d \xi}\right|_{\xi=0}$; is whole number or fraction, belongs [0,2];

$\mathrm{N}$ from $\rightarrow \mathrm{m}$ (see Table 1.)

Core Criterion: $f(m)=\left.\frac{d \theta}{d \xi}\right|_{\xi=1}$; is whole number or fraction, belongs $[0, \pm \infty]$;

$f(m)$ from $\rightarrow \mathrm{m}$ (see Table 1.).

$c=f_{1}(N)$ and $n=f_{2}(N)$ : changeable interconnected coefficient and exponent; whole numbers or fractions.

$\mathrm{m}$ : the whole characteristic number, presents the ratio of formation $(\mathrm{F})$ and decomposition (D) processes inside the laminar boundary layer, $\mathrm{m}=\mathrm{F} / \mathrm{D}=1,2,3 \ldots$

The quantity m enables the total coupling of the analyzed situation.

Introducing the relevant notation

for steady state flow: $\theta, \xi=\mathrm{y} / \delta_{\chi}$ where $\delta_{\chi}=C_{\chi} \sqrt{\frac{\Pi \chi}{\vartheta_{\xi=1}}}$

( $\mathrm{C}_{\chi}$ : coefficient of proportionality).

- for unsteady state flow/non flow: $\Theta=1-\theta, \xi=\mathrm{z} / \delta_{\tau}$ where $\delta_{\tau}=C_{\tau} \sqrt{\Pi \tau}$

$\left(\mathrm{C}_{\tau}\right.$ : coefficient of proportionality).

the solution of equation (1) becomes the polynomial

for steady state flow: $\theta=N \xi \pm \frac{1}{2} f(m) \xi^{2} \pm(N-1) \xi^{\left[\frac{N}{ \pm(N-1)}\right]^{( \pm 1) *}}$

- for unsteady state flow/non flow: $\Theta=1-\theta$

Normalized distribution $\theta$ (or $\Theta$ ), a solution of the equation (1), has similar meaning for velocity, temperature or concentration. 
Steady state flow solution

For steady state flow, when $f(\mathrm{~m})=0$, the criterion $N=\left.\frac{d \theta}{d \xi}\right|_{\xi=0}$ defines the state at the cross-section perpendicular to the surface. This further enables the comparison and classification of typical flows with:

reference distribution

$\theta_{\mathrm{N}=1}=\xi$ : simple Coutte flow

and boundary distributions

lower: $\theta \mathrm{N}=0=1$ : free flow and

upper: $\theta_{\mathrm{N}=2}=2 \xi-\xi^{2}$ : quadratic Poiseuille flow.

Here, the solutions of eq. 1. are families of polynomial curves grouped in the four regions: $[0 ; 1 / 2],[1 / 2 ; 1],[1 ; 3 / 2]$ and $[3 / 2 ; 2]$ with appropriate $\mathrm{N}$ and $f(\mathrm{~m})$ whole numbers and/or fractions are given in Table 1 .

Fig. 2 illustrates the existence of two types of distributions in the region $[0 ; 1 / 2]$ : „blade“ and quasi-laminar.

Table 1. Elements of the solution for steady state flow: $\theta=\left(v-v_{0}\right) /\left(v_{\xi=1}-v_{0}\right)$ and $\xi=y / \delta_{\chi}$, where $\vartheta$ denotes velosity.

\begin{tabular}{|l|l|}
\hline Ordinary differential equation: & $\theta^{\prime \prime}(\xi) \pm c \xi^{n} \pm f(m)=0$ \\
\hline General solution: & $\theta=N \xi \pm \frac{1}{2} f(m) \xi^{2} \pm(N-1) \xi^{\left[\frac{N}{ \pm(N-1)}\right]^{( \pm 1) *}}$ \\
\hline When $f(\mathrm{~m})=0$ & \\
\hline Reference distribution: & $\theta_{\mathrm{N}=1}=\xi:$ simple Coutte flow \\
\hline Boundary distributions: & \\
\hline Lower: & $\begin{array}{l}\theta_{\mathrm{N}=0}=1: \text { free flow } \\
\theta_{\mathrm{N}=2}=2 \xi-\xi^{2}: \text { quadratic Poiseuille flow }\end{array}$ \\
\hline
\end{tabular}




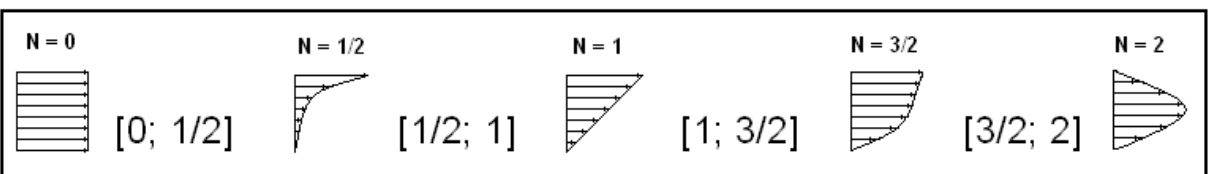

\begin{tabular}{|c|c|c|c|c|}
\hline Regions of $\mathrm{N}$ & {$[0 ; 1 / 2]$} & {$[1 / 2 ; 1]$} & {$[1 ; 3 / 2]$} & {$[3 / 2 ; 2]$} \\
\hline $\mathrm{N}$ & $1-m /(m+1)$ & $\mathrm{m} /(\mathrm{m}+1)$ & $2-m /(m+1)$ & $1+\mathrm{m} /(\mathrm{m}+1)$ \\
\hline$f(\mathrm{~m})$ & $2-1 / \mathrm{m}$ & $2-m$ & $\mathrm{~m}$ & $1 / \mathrm{m}$ \\
\hline $\begin{array}{l}{[\mathrm{N} / \pm(\mathrm{N}-1)]^{( \pm 1)}} \\
(\mathrm{N}-1) \xi^{[\mathrm{N} / \pm(\mathrm{N}-1)]^{( \pm 1)^{*}}}\end{array}$ & $\begin{array}{l}{[-(\mathrm{N}-1) / \mathrm{N}] \quad \boldsymbol{q} \boldsymbol{L}^{*}} \\
0[\mathrm{f}(\mathrm{m})=0] \quad \boldsymbol{b}^{*}\end{array}$ & {$[\mathrm{~N} /-(\mathrm{N}-1)]$} & {$[\mathrm{N} /(\mathrm{N}-1)]$} & {$[\mathrm{N} /(\mathrm{N}-1)]$} \\
\hline B.C. & $\xi=0 \Rightarrow \theta=0$ & $\xi=0 \Rightarrow \theta=0$ & $\xi=0 \Rightarrow \theta=0$ & $\xi=0 \Rightarrow \theta=0$ \\
\hline when $f(\mathrm{~m})=0$ : & $\xi=1 \Rightarrow \theta=1$ & $\xi=1 \Rightarrow \theta=1$ & $\xi=1 \Rightarrow \theta=1$ & $\xi=1 \Rightarrow \theta=1$ \\
\hline when $f(\mathrm{~m}) \neq 0$ : & $\begin{array}{c}(\xi=1 \Rightarrow \theta \neq 1 \\
\left.b^{*}\right) \\
\xi=1 \Rightarrow \theta \neq 1\end{array}$ & $\xi=1 \Rightarrow \theta \neq 1$ & $\xi=1 \Rightarrow \theta \neq 1$ & $\xi=1 \Rightarrow \theta \neq 1$ \\
\hline $\begin{array}{l}\theta(\text { when } \mathrm{m}=2 ; \\
f(\mathrm{~m})=0)\end{array}$ & $\begin{array}{c}1 / 3 \xi+2 / 3 \xi^{2} \\
\boldsymbol{q} \boldsymbol{L}^{*} \\
1 / 3 \xi \boldsymbol{b}^{*} \\
\end{array}$ & $2 / 3 \xi+1 / 3 \xi^{2}$ & $4 / 3 \xi-1 / 3 \xi^{4}$ & $5 / 3 \xi-2 / 3 \xi^{5 / 2}$ \\
\hline $\begin{array}{l}\mathrm{d} \theta /\left.\mathrm{d} \xi\right|_{\xi=0} \\
(\text { when } \mathrm{m}=2 ; \\
f(\mathrm{~m})=0)\end{array}$ & $\begin{array}{cc}\mathrm{N}=1 / 3 & \left(\boldsymbol{q} \boldsymbol{L}^{*}\right) \\
\mathrm{N}=1 / 3 & \left(\boldsymbol{b}^{*}\right)\end{array}$ & $\mathrm{N}=2 / 3$ & $\mathrm{~N}=4 / 3$ & $N=5 / 3$ \\
\hline $\mathrm{d} \theta /\left.\mathrm{d} \xi\right|_{\xi=1, f(\mathrm{~m})=0}$ & $\neq 0$ & 0 & 0 & 0 \\
\hline $\mathrm{d} \theta /\left.\mathrm{d} \xi\right|_{\xi=1, f(\mathrm{~m}) \neq 0}$ & $\neq 0$ & $\neq 0$ & $\neq 0$ & $\neq 0$ \\
\hline
\end{tabular}

$\boldsymbol{q} \boldsymbol{L}^{*}$ : quasi-Laminar; $\boldsymbol{b}^{*}$ : "blade" 


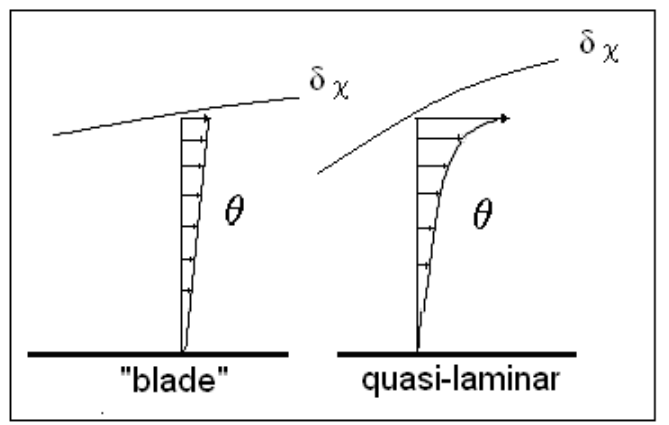

Fig. 2. Schematic of "blade" and quasi-laminar distributions.

Coding of laminar boundary layers

The Flux Gradient (FG-code) [4-7] of the quantity $\vartheta$ change inside laminar boundary layer, can be defined, from the equation (1), as:

$$
F G=\int_{1}^{0} \theta^{\prime \prime}(\xi) d \xi=\left.\frac{d \theta}{d \xi}\right|_{\xi=0}-\left.\frac{d \theta}{d \xi}\right|_{\xi=1}=N \pm f(m)
$$

Note: $\mathbf{F G} \equiv \mathbf{N}$ when $f(\mathrm{~m})=0$

Generally, two forms of laminar layers are possible, when:

(a) FG is constant along the referent axis, (examples of the layers over a flat plate and rotating disk) and

(b) FG is variable, (an example of laminar entrance region flow). Every position of $\chi$ has its corresponding distribution.

Analogous coding for unsteady state boundary layers is also possible.

\section{Verification}

The example of exact solutions for simultaneous heat, mass and momentum transfer according to boundary layer theory [7] is used as a confirmation of this concept.

Example. The sharp-edged semi-infinite flat plate of volatile solid A sublimes, into the tangential flow of the gaseous stream of A and B. We have mass transfer into the stream under steady conditions, knowing that species B is presented only in the gas phase. For the uniform temperature and gas composition along the plate surface, we must determine the profiles of velocity, temperature, and concentration in the laminar boundary layer. (see Fig. 1, steady state flow, Blasius eq.)

The boundary layer equation over a flat plate is:

$$
-\Lambda \mathrm{f} \theta^{\prime}=\theta^{\prime \prime}
$$


The boundary conditions, when $\xi=\frac{y}{\delta_{\chi}}=\frac{y}{2} \sqrt{\frac{v_{\infty}}{v x}}$, are:

at $\xi=0, \theta=0$

at $\xi=\infty, \theta=1$

The dimensionless profile variables are:

$\theta_{u}=\frac{v_{x}-v_{x 0}}{v_{x \infty}-v_{x 0}}=\frac{v_{x}}{v_{\infty}} \quad \theta_{T}=\frac{T-T_{0}}{T_{\infty}-T_{0}} \quad \theta_{A B}=\frac{x_{A}-x_{A 0}}{x_{A \infty}-x_{A 0}}$

The dimensionless physical property ratios are:

$\Lambda_{v}=\frac{v}{v}=1 \quad \Lambda_{T}=\frac{v}{\alpha}=\operatorname{Pr} \quad \Lambda_{A B}=\frac{v}{D_{A B}}=S c$

The quantity $\mathrm{f}$ is:

$\mathrm{f}=-\mathrm{K}+\int_{0}^{\xi} 2 \theta_{u} d \xi$

The $\mathrm{K}$ is dimensionless mass flux at the wall:

$K=\frac{1}{\Lambda_{A B}}\left(\frac{x_{A 0}-x_{A \infty}}{1-x_{A 0}}\right) \theta_{A B}^{\prime} d \xi$

Note: $\mathrm{K}$ is analogous to $\mathrm{FG}$ or $\mathrm{N}$ when $f(\mathrm{~m})=0$.

In the literature the "exact solution" of Eq. (4), denotes a power series solution of the Blasius as opposed to more approximate solutions, i.e., polynomials of von Karman or Schlichting. Overview of commonly used solutions in addition with a proposed solution when $\mathrm{m}=\mathrm{F} / \mathrm{D}=2$ and $f(m)=0$, is presented in Fig. 2.

Table 2. Overview of commonly used equations when $\mathrm{K}=0$.

\begin{tabular}{|l|l|l|}
\hline$\Theta$ & $\left.\frac{d \theta}{d \xi}\right|_{\xi=0}$ & $\delta_{\chi} \sqrt{\frac{v_{\infty}}{v x}}$ \\
\hline Blasius & 1.66 & 5.00 \\
\hline von Karman, $\frac{3}{2} \xi-\frac{1}{2} \xi^{*}$ & $3 / 2$ & 4.64 \\
\hline $\sin \left(\frac{\pi}{2} \xi\right)$ & $\pi / 2$ & 4.80 \\
\hline Schlichting, $2 \xi-2 \xi^{3}+\xi^{4}$ & 2 & 5.83 \\
\hline$\xi$ & 1 & 3.46 \\
\hline $\begin{array}{l}\text { Proposed equation, } \\
\frac{5}{3} \xi-\frac{2}{3} \xi^{\frac{5}{2}}, \mathrm{~m}=\mathrm{F} / \mathrm{D}=2 \text { and } f(m)=0\end{array}$ & $5 / 3$ & 4.94 \\
\hline
\end{tabular}

* same as proposed method, when $\mathrm{m}=\mathrm{F} / \mathrm{D}=1$ and $f(m)=0$ 
Comparison of results calculated from laminar boundary layer theory [7] and the proposed method for $2 \theta^{\prime}(0, \Lambda, K)$ and $\theta^{\prime}(0, \Lambda, 0)$ are given in Tables 3 and 4, respectively.

Table 3. Dimensionless gradients of velocity, temperature, and composition calculated by ${ }^{(a, b, c, d, e)}[7]$ and the proposed method.

Dimensionless gradient $2 \theta^{\prime}(0, \Lambda, \mathrm{K})$ at the flow boundary.

\begin{tabular}{|l|l|l|l|l|l|l|}
\hline $\mathrm{K}$ & $\mathbf{( N )}$ & $\Lambda=0.8$ & $\Lambda=0.9$ & $\Lambda=1.0$ & $(\Lambda=\mathbf{1 . 0})$ & $\Lambda=1.1$ \\
\hline $1.0^{(\mathrm{e})}$ & $\mathbf{( 4 / 3 )}$ & 0.1831 & 0.1615 & 0.1419 & $\mathbf{( 0 . 1 4 3 7 )}$ & 0.1242 \\
\hline $0.5^{(\mathrm{e})}$ & & 0.6649 & 0.6626 & 0.6580 & & 0.6516 \\
\hline $0.0^{(\mathrm{c})}$ & $\mathbf{( 5 / 3 )}$ & 1.280 & 1.280 & $1.328^{(\mathrm{b})}$ & $\mathbf{( 1 . 3 4 9 )}$ & 1.375 \\
\hline$-0.5^{(\mathrm{d})}$ & & 1.970 & 1.970 & 2.092 & & 2.213 \\
\hline$-1.0^{\text {(d) }}$ & $\mathbf{( 2 / 3 )}$ & 2.709 & 2.709 & 2.916 & $\mathbf{( 3 . 2 4 4 )}$ & 3.122 \\
\hline$-5.0^{\text {(d) }}$ & $\mathbf{( 1 / 3 )}$ & 8.388 & 9.375 & 10.36 & $\mathbf{( 9 . 9 3 9 )}$ & 11.35 \\
\hline
\end{tabular}

( ) Proposed method, when $\mathrm{m}=\mathrm{F} / \mathrm{D}=2$ and $f(m)=0$

Table 4. Comparison of results for the profile gradients at negligible mass transfer rates [7] and the proposed method.

Predicted values of $\theta^{\prime}(0, \Lambda, 0)$

\begin{tabular}{|l|l|l|l|l|}
\hline$\Lambda$ & Exact & $\begin{array}{l}\text { Asymptotic } \\
\text { solution }\end{array}$ & $\begin{array}{l}\text { Pohlhausen's } \\
\text { approx. }\end{array}$ & $\begin{array}{l}\text { Proposed method } \\
\text { when } \mathrm{m}=2 \text { and } f(m)=0\end{array}$ \\
\hline 0.6 & 0.552 & 0.571 & 0.560 & 0.568 \\
\hline 0.8 & 0.614 & 0.629 & 0.616 & 0.626 \\
\hline 1.0 & 0.664 & 0.677 & 0.664 & 0.674 \\
\hline 2.0 & 0.845 & 0.853 & 0.837 & 0.849 \\
\hline
\end{tabular}

Also, this concept has been experimentally verified in the field of materials science and engineering: gas carburizing [8], CVD processes [9], phenomena of external and internal surfaces of micro solids [10], diffusion into filler metal [11], dislocations flow [12], hillocks growth [13] etc.

\section{Concluding remarks}

Simulation of multi-dimensional simultaneous transport phenomena under laminar conditions needs a considerable computation time often with faulty assumptions. Contrary, expensive and long-term experimental validation is not always a good confirmation for numerical results. Consequently, the new, much more lifelike and realistic simulation strategy, presented in Fig. 3 is suggested. 


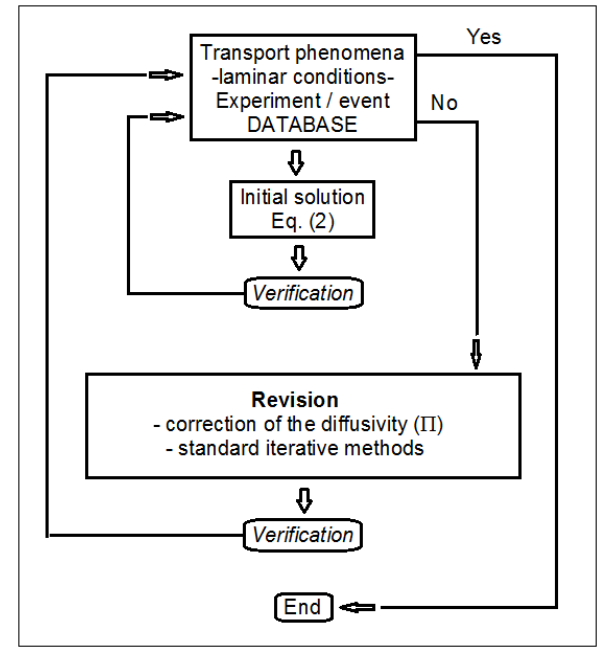

Fig. 3. Flowchart of the simulation strategy.

Exact data of starting experiment or event give the "real" distribution $(\phi)$ as opposite to an approximate distribution $(\theta)$, eq. (2). When necessary, the difference $(\phi-$ $\theta$ ) could be minimized by: (a) continuous correction of the diffusivity ( $\Pi$ ) when $\Pi=f(\vartheta, \xi)[14]$ and/or (b) standard iterative methods [15].

Presented flexible, iterative system for the following experimental events needs further numerical improvement and modification.

\section{Acknowledgments}

This work was financially supported by the Ministry of Education, Science and Technological Development of the Republic of Serbia, grant number 172005.

\section{References}

[1] M. R. Mobinipouya, M. M. Papari, In: Proceedings title. Second International Conference on Environmental and Computer Science, 2009, 356-359.

[2] S. Makhlouf, M. Laghrouche, Abd El Hamid Adane: IEEE Sens J, 16 (2016) 26502657.

[3] M. Mustafa, Junaid A. Khan, T. Hayat, A. Alsaedi: Trans Nanotechnol, 14 (2015) 159-168.

[4] K. Parand, Mehdi Dehghan, F. Baharifard: Applied Mathematical Modelling, 37 (2013) 851-863.

[5] K. Stewartson: J Math Phys: 36, 1-4 (1957) 173-191.

[6] H. Schlichting \& K. Gersten, Boundary Layer Theory, 8th ed., Springer, 2000, pp. 145-207 and 683-703.

[7] R.B. Bird, W.E. Stewart and E.D. Lightfoot, Transport Phenomena, John Wiley and Sons, New York, 1960, pp.608-619.

[8] K.T. Raic: ISIJ Int, 32 (1992) 514-520.

[9] K.T. Raic: Le Journal de Physique IV, C5 (1995) 235-242.

[10] K.T. Raic: Interface Sci, 8 (2000) 85-94. 
[11] K.T. Raic: Ceram Int, 26, 1, (2000) 19-24.

[12] K.T. Raic: Sci Technol Adv Mater, 9 (2008) 015008.

[13] K.T. Raic: Surf Eng, 32, 11 (2016) 823-828.

[14] S.V. Patankar, Numerical Heat Transfer and Fluid Flow, Hemisphere Publ Corp, New York, 1980, pp.54-61.

[15] C. T. Kelley, Iterative Methods for Linear and Nonlinear Equations, Society for Industrial and Applied Mathematics, Philadelphia, 1995.

\section{Nomenclature}

a thermal diffusivity

C concentration

D diffusivity or diffusion coefficient throughout the layer

$D_{A B} \quad$ diffusion coefficient of $A$ into an unbounded gaseous stream of $A$ and $B$

FG Flux Gradient (FG-code)

$\mathrm{K}$ dimensionless mass flux at the wall

$\mathrm{m} \quad$ characteristic whole number

$\mathrm{N}$ dimensionless criterion

$\mathrm{T}$ temperature

$\mathrm{XA} \quad$ concentration

$\mathrm{x} ; \mathrm{y} ; \mathrm{z}$ coordinats

Greek symbols

$$
\begin{aligned}
& \mathrm{A} \\
& \delta_{\chi} \\
& \delta_{\tau} \\
& \xi=\mathrm{y} / \delta_{\chi} \text { or } \xi=\mathrm{z} / \delta_{\tau} \\
& \nu \\
& \Pi \\
& \theta=\frac{\vartheta-\vartheta_{0}}{\vartheta_{\xi=1}-\vartheta_{0}} \\
& \theta^{\prime}=\mathrm{d} \theta / \mathrm{d} \xi \\
& \theta^{\prime \prime}=\mathrm{d}^{2} \theta / \mathrm{d} \xi^{2} \\
& \Theta=1-\theta \\
& \Lambda_{v}=\frac{v}{v}=1 \\
& \Lambda_{T}=\frac{v}{\alpha}=\operatorname{Pr} \\
& \Lambda_{A B}=\frac{v}{D_{A B}}=S c
\end{aligned}
$$$$
\text { v }
$$$$
\text { thermal diffusivity }
$$$$
\text { boundary layer thickness at position }(\chi)
$$$$
\text { boundary layer thickness at time }(\tau)
$$$$
\text { dimensionless distance from sur/interface for position } \chi \text { or time } \tau
$$$$
\text { kinematic viscosity }
$$$$
\text { viscosity/diffusivity }(v, a, D)
$$$$
\text { normalized dimensionless change of quantity } \vartheta \text { (velocity, } v \text {; }
$$$$
\text { temperature, } \mathrm{T} \text {; and/or concentration, } \mathrm{C} \text { or } \mathrm{x}_{\mathrm{A}} \text { ) }
$$$$
\text { derivation of first order }
$$$$
\text { derivation of second order }
$$$$
\text { normalized concentration distribution at time }(\tau) \text { for un-steady state }
$$$$
\text { flow/non-flow }
$$

Prandtl number

Schmidt number

velocity

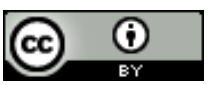

Creative Commons License

This work is licensed under a Creative Commons Attribution 4.0 International License. 